+ and from Day 2 (median 6; 2-19), 5/20 were CMV+ (p = 0.96). Median cumulative steroid dose prior to biopsy was $0.28 \mathrm{~g}$ prednisolone equivalent for $\mathrm{CMV}+$ patients and $0.39 \mathrm{~g}$ for CMVpatients $(\mathrm{p}=0.51) .3 / 13 \mathrm{CMV}+$ patients had had prednisolone $\geq 10 \mathrm{mg} /$ day for $>14$ days prior to biopsy and 11/32 CMVpatients $(p=0.46)$. No biopsies contained typical histological features of CMV infection. Mayo and Baron scores did not differ between CMV+ and CMV- patients. Mucosal CMV DNA titre in $\mathrm{CMV}+$ patients did not correlate with Baron or Mayo scores. Of the mucosal CMV+ patients, $8 / 8$ tested $(100 \%)$ were anti-CMV IgG positive with $0 / 7$ IgM positive. Peripheral blood CMV PCR was positive in 10/13 in the biopsy PCR + group and 0/7 in those biopsy PCR- (sensitivity 60\%, specificity 100\%). 12/13 CMV PCR + patients were treated with ganciclovir. 11/13 (84.6\%) achieved remission. 2/13 (15.3\%) CMV+ and 2/32 (6.2\%) CMV- required colectomy $(\mathrm{p}=0.33)$. All 6 patients treated concomitantly with Infliximab and ganciclovir responded fully.

Conclusion PCR of mucosal biopsies detects CMV infection due to viral reactivation in almost a third of patients with deteriorating or acute severe UC. No relationship was demonstrated between CMV infection and disease severity, response to treatment or prior steroid use. Treatment with anti-TNF agents was administered safely in combination with anti-viral drugs. Disclosure of Interest None Declared.

\section{PTU-084 PREVENTION OF OPPORTUNISTIC INFECTIONS IN PATIENTS ON BIOLOGICAL AGENTS FOR MANAGEMENT OF INFLAMMATORY BOWEL DISEASE}

H Gordon*, A Steel. Gastroenterology, Chelsea and Westminster Hospital, London, UK

\subsection{6/gutjnl-2014-307263.158}

Introduction Patients with inflammatory bowel disease are at increased risk of infection; this is especially true of the $20 \%$ on biological agents. ECCO guidelines recommend the following vaccines:

Influenza (annual), Pneumococcal, Hepatitis B, Varicella, HPV (women under 26).

The guidelines also highlight the need to exclude latent TB; local policy is to perform an interferon gamma release assay.

Within the UK vaccination services are provided by primary care.

Methods The measures taken to prevent opportunistic infection in patients prescribed anti-TNFs for IBD at Chelsea and Westminster Hospital in 2013 were audited against the ECCO OI Guidelines.

The following were retrieved from electronic records: age, sex, anti TNF prescribed, pneumococcal antibodies, hepatitis B core and surface antibodies, varicella $\operatorname{IgG}$, Elispot ${ }^{\circledR}$.

Attempts were made to retrieve vaccination history from General Practice.

Results 60 patients were prescribed infliximab and 15 patients were prescribed adalimumab. 46 GPs were able to provide vaccination history.

Influenza: $50 \%(23 / 46)$ patients received vaccination against influenza within the past year.

Pneumococcus: $55 \%(47 / 85)$ patients demonstrated immunity. $6 \%(5 / 85)$ were not immune and the remainder were not tested. The vaccination history of 26 patients who were not immune or not tested was retrieved. 27\% (7/26) had since been vaccinated.

Hepatitis B: No patients were core Ab positive. Surface Ab levels demonstrated immunity in 7\% (6/85). 53\% (45/85) were not immune, and the remainder were not tested. Vaccination history of 44 patients who were not immune or not tested was retrieved. Of these, $25 \%(11 / 44)$ had since been vaccinated.

HPV: 4 patients were women under 26 years old. $25 \%(1 / 4)$ had confirmed HPV vaccination.

Varicella: $21 \%(18 / 85)$ patients demonstrated immunity to varicella. $2 \% 2 / 85$ were not immune.

Elispot: $65 \%(55 / 85)$ patients had a nonreactive assay. 1\% (1/ 85 ) had a positive result and the remainder were not tested.

Conclusion The standards set out by ECCO to protect patients from opportunistic infection are not being met.

Problems obtaining accurate vaccination history from GP records include incorrect surgery details, lack of availability of staff able to review records and incomplete records. HPV vaccination usually takes place at school and is not routinely recorded by primary care.

Potential service improvements include provision of vaccines at clinic, improved patient education regarding the importance of vaccination and a check list to review bloods at first anti-TNF prescription.

\section{REFERENCE}

1 Rahier JF, Ben-Horin S, Chowers $Y$, Conlon C, De Munter P, D'Haens G, Domènech E, Eliakim R, Eser A, Frater J, et al. European evidence-based Consensus on the prevention, diagnosis and management of opportunistic infections in inflammatory bowel disease. J Crohns Colitis 2009;3:47-91

Disclosure of Interest None Declared.

\section{PTU-085 DID THE 2010 NICE GUIDELINES IMPACT ON BIOLOGICS PRESCRIPTION?}

${ }^{1}$ H Steed ${ }^{*},{ }^{2}$ A Townsend, ${ }^{1}$ A Rowlinson, ${ }^{1} \mathrm{~F}$ Leslie. 'Gastroenterology, University Hospital North Staffordshire, UK; ${ }^{2}$ Medical School, Keele University, Stoke-on-Trent, UK

\subsection{6/gutjnl-2014-307263.159}

Introduction In 2010 NICE updated their guidelines on the prescription of anti-tumour necrosis factor alpha (a-TNF) for Crohn's disease (CD) to include Adalimumab as a treatment for patients with severe active CD. As a therapeutic option it provided an attractive alternative, both to units, struggling for infusion capacity and limited specialist nurse input, and to patients, as a time-saving, home delivered and administered option.

Methods A retrospective review of inflammatory bowel disease patients at University Hospital North Staffordshire up until December 2012.

Results Of 3600 inflammatory bowel disease 117 patients are on a-TNF. A further 47 patients have had, but are no longer on, a-TNF. 37 patients had their original a-TNF switched during their disease course (21\%). 25 patients (68\% of all switches) had their a-TNF switched for non-clinical reasons ("including patient choice and unit preference). All of these were from infliximab to adalimumab, 8 had to be switched back to infliximab.

\begin{tabular}{ll} 
Abstract PTU-085 Table 1 & \\
\hline Year & No of non-clinical switches \\
\hline 2008 & 3 \\
2009 & 0 \\
2010 & 15 \\
2011 & 3 \\
2012 & 4 \\
\hline
\end{tabular}

